\title{
Densidade de estocagem na engorda de camarão-branco cultivado em sistema de biofloco
}

\author{
Charles Fróes ${ }^{(1)}$, Geraldo Fóes( ${ }^{(1)}$, Dariano Krummenauer ${ }^{(1)}$, Luis Henrique Poersch ${ }^{(1)}$ e Wilson Wasielesky Junior ${ }^{(1)}$ \\ (1)Universidade Federal do Rio Grande, Instituto de Oceanografia, Estação Marinha de Aquacultura, Rua do Hotel, 02, Cassino, \\ CEP 96210-030 Rio Grande, RS. E-mail: charlesfroes@gmail.com, geraldofoes@gmail.com, darianok@gmail.com, Ipoersch@mikrus.com.br, \\ manow@mikrus.com.br
}

Resumo - O objetivo deste trabalho foi determinar a densidade de estocagem ideal para o cultivo de camarão-branco, em sistema de biofloco sem renovação de água. Foram realizados dois experimentos inteiramente casualizados, com três repetições para cada tratamento. No primeiro, juvenis de camarão-branco, com peso médio inicial de $1,23 \pm 0,09 \mathrm{~g}$, foram cultivados a densidades de estocagem de 833, 1.250, 1.667, 2.083 e 2.500 camarões por $\mathrm{m}^{3}$. No segundo experimento, o peso médio inicial foi de $6,32 \pm 0,7 \mathrm{~g}$, e as densidades foram de 566, 833, 1.111, 1.388 e 1.667 camarões por $\mathrm{m}^{3}$. Os melhores resultados de produtividade, com médias de 9,92 e $10,77 \mathrm{~kg} \mathrm{~m}^{-3}$, foram obtidos com 1.667 e 1.111 camarões por $\mathrm{m}^{3}$, respectivamente, no primeiro e segundo experimentos. $\mathrm{O}$ aumento da densidade de estocagem afeta o crescimento e a sobrevivência do camarão-branco em sistema de biofloco.

Termos para indexação: Litopenaeus vannamei, desempenho, produtividade, renovação de água.

\section{Stocking density at rearing phase for white shrimp farmed in a biofloc system}

\begin{abstract}
The objective of this work was to determine the optimal stocking density for Pacific white shrimp farming, in biofloc culture system (BFT) without water exchange. Two completely randomized experiments were carried out, with three replicates for each treatment. In the first experiment, juveniles with $1.23 \pm 0.09 \mathrm{~g}$ initial mean weight were reared at the stocking densities of $833,1,250,1,667,2,083$, and 2,500 shrimp per $\mathrm{m}^{3}$. In the second experiment, the initial mean weight was $6.32 \pm 0,7 \mathrm{~g}$, and the densities were 566 , $833,1,111,1,388$, and 1,667 shrimps per $\mathrm{m}^{3}$. The best productivity results, with the means 9.92 and 10.77 $\mathrm{kg} \mathrm{m}^{-3}$, were obtained at 1,667 and 1,111 shrimp per $\mathrm{m}^{3}$, respectively, in the first and second experiments. Increasing stocking density affects growth and survival of white shrimp farmed in biofloc system.
\end{abstract}

Index terms: Litopenaeus vannamei, performance, productivity, water renovation.

\section{Introdução}

A densidade de estocagem tem grande importância na produtividade das fazendas de camarão marinho, onde muitos produtores optam por intensificar os cultivos com o objetivo de minimizar os custos e garantir a sustentabilidade econômica. Entretanto, esta intensificação gera maior dependência de aeração artificial, mão de obra especializada e dietas comerciais com elevados níveis de proteínas (Decamp et al., 2007). Além disto, cultivos intensivos de camarões utilizam altas taxas de renovação de água para garantir a qualidade desta, o que gera efluentes com elevadas concentrações de nutrientes e matéria orgânica (Boyd \& Clay, 1998), com a possibilidade de eutrofização de ambientes aquáticos adjacentes.

O sistema de biofloco sem renovação de água surge como alternativa para aumentar a produtividade das fazendas e diminuir seu impacto ambiental. Neste sistema, é estimulada a formação dos chamados flocos microbianos, compostos por bactérias, flagelados, ciliados, cianobactérias, microalgas e pequenos metazoários, além de detritos orgânicos (Wasielesky Junior et al., 2006). Os viveiros são altamente oxigenados e fertilizados com fontes ricas em carbono, para estimular o surgimento de uma biota bacteriana predominantemente heterotrófica (Avnimelech, 2009).

As bactérias presentes no sistema biofloco assimilam os compostos nitrogenados dissolvidos na água de cultivo, gerados principalmente pela excreção e restos de alimentos em decomposição, o que possibilita que a água seja reutilizada em diversos ciclos (Avnimelech, 2009). Ademais, os microrganismos do biofloco têm a capacidade de reciclar os compostos nitrogenados, tornando-se uma rica fonte proteica para os organismos cultivados neste sistema (Wasielesky Junior et al., 
2006). Assim, poderá haver redução da utilização de farinha de peixe como fonte principal de proteína (Avnimelech, 2009). Outra vantagem deste sistema de cultivo é o maior grau de biossegurança, por não haver troca de água com o ambiente aquático adjacente, e pela redução do risco de introdução e disseminação de patógenos (Wasielesky Junior et al., 2006).

Para garantir a viabilidade econômica do sistema de biofloco, é necessária uma densidade de estocagem acima de 1.111 camarões por metro cúbico (Otoshi et al., 2007). Porém, há uma relação negativa entre o aumento da densidade e o desempenho zootécnico dos camarões (Moss \& Moss, 2004; Arnold et al., 2009). A redução do crescimento e da sobrevivência a altas densidades de estocagem é resultado da diminuição de espaço, do aumento de canibalismo, da competição por alimento natural, da concorrência por espaço, da degradação da qualidade da água e do acúmulo de sedimentos indesejáveis no fundo dos viveiros (Krummenauer et al., 2006, 2011).

As densidades de estocagem ideais a serem utilizadas variam muito, a depender da espécie, fase de vida, sistema de cultivo e práticas de manejo empregadas em cada fazenda. Em razão do alto custo de construção e operação dos sistemas de biofloco (Boyd \& Clay, 1998; Wasielesky Junior et al., 2006), é importante segmentar a fase de engorda do camarão-branco, a fim de utilizar-se ao máximo o espaço disponível, e melhorar as técnicas de manejo.

O objetivo deste trabalho foi determinar a densidade de estocagem ideal para o cultivo de camarão-branco, em sistema de biofloco sem renovação de água.

\section{Material e Métodos}

Os experimentos foram realizados na Estação Marinha de Aquacultura Professor Marcos Alberto Marchiori, do Instituto de Oceanografia, da Universidade Federal do Rio Grande (Ema/IO/Furg), situada na cidade do Rio Grande, RS, de 11 de janeiro a 3 de abril de 2010.

Paraavaliarapenas os efeitos do estresse populacional sobre o desempenho zootécnico dos camarões, montouse uma estufa fechada com sistema de recirculação de água, que continha 15 tanques (microcosmo), com área de fundo de $0,5 \mathrm{~m}^{2}$ e capacidade de $180 \mathrm{~L}$, supridos por aeração intensa e individual, com uma saída de água por gravidade para uma calha que a direcionava até um tanque-matriz (macrocosmo) em sistema "raceway" de $70 \mathrm{~m}^{2}$, onde o suprimento de ar e circulação da água foram feitos através de um soprador de ar ("blower"). A água era bombeada novamente para as unidades experimentais, à vazão de $6,6 \mathrm{~L} \mathrm{~min}^{-1}$ por tanque. Com o objetivo de garantir a mesma qualidade ambiental em todos a unidades experimentais, a taxa de renovação diária era 53 vezes a do volume de água total. No tanque-macrocosmo, em sistema de biofloco sem renovação de água, foi mantido um cultivo de camarão-branco com peso médio inicial de $3 \pm 0,6 \mathrm{~g}$ e, à densidade de estocagem de 300 camarões por metro cúbico. Ao final dos experimentos, os camarões do tanque-matriz apresentavam peso médio de $16 \pm 1,2 \mathrm{~g}$.

Com o objetivo de estimular o crescimento da população bacteriana e, consequentemente, o aumento da biomassa microbiana (biofloco), o sistema foi fertilizado com melaço à razão de $6 \mathrm{mg}$ de carbono para cada $1 \mathrm{mg}$ de amônia total presente na água (Avnimelech, 2009). Para manter os níveis adequados de $\mathrm{pH}$, adicionou-se carbonato de sódio $\mathrm{Ph}+$, (Hidro Ltda., Valinhos, SP), conforme proposto por Furtado et al. (2011).

Para avaliar a densidade de estocagem ideal, nas diferentes fases de engorda do camarão-branco, foram realizados sequencialmente dois experimentos, em delineamento inteiramente casualizado, com três repetições.

No experimento I, juvenis de camarão com peso médio inicial de $1,23 \pm 0,09 \mathrm{~g}$ foram cultivados por 40 dias às densidades de 833, 1.250, 1.667, $2.083 \mathrm{e}$ 2.500 camarões por metro cúbico. No experimento II, durante 40 dias, camarões com peso médio inicial de $6,32 \pm 0,7 \mathrm{~g}$ foram cultivados às densidades de $556,833,1.111,1.388$ e 1.667 camarões por metro cúbico.

As determinações de temperatura, $\mathrm{pH}$ e oxigênio dissolvido foram realizadas diariamente em cada unidade experimental (microcosmo), por meio de um multiparâmetro modelo 556 MPS (YSI Inc., Yellow Springs, OH, EUA). As concentrações de amônia total, nitrito, nitrato e fosfato foram medidas a cada três dias, conforme Baumgarten et al. (2010). As coletas de água para as análises de salinidade e nutrientes foram feitas no tanque-matriz (macrocosmo).

Os camarões foram alimentados duas vezes ao dia (às 10 e às $18 \mathrm{~h}$ ), com a ração comercial Active 38 (Centro Oeste Rações Ltda., Guabi, Campinas, SP), composta 
por $38 \%$ proteína bruta. A taxa diária de arraçoamento inicial foi de $5 \%$ da biomassa de camarões, que foi ajustada de acordo com o consumo.

No início dos experimentos, a obtenção do peso médio inicial (úmido) foi feita a partir de 200 camarões, selecionados ao acaso, secos individualmente em papel-toalha e pesados em balança de precisão Ohaus Adventurer Pro (Toledo do Brasil, Barueri, SP). Após o início dos experimentos, 50 indivíduos de cada unidade experimental, foram pesados a cada 10 dias. Após a pesagem, os camarões foram devolvidos à unidade experimental de origem.

Depois de verificada a homocedasticidade e normalidade, os dados foram submetidos a análise de variância univariada e, posteriormente, ao teste de Tukey, a 5\% de probabilidade (Sokal \& Rohlf, 1969). Em caso de significância estatística (a 5\% de probabilidade), foram ajustados alternativamente os modelos de regressão linear ou quadrático para as variáveis de resposta, em razão das diferentes densidades de estocagem. Os dados de percentagem passaram pela transformação arcosseno antes da análise.

\section{Resultados e Discussão}

Os parâmetros de qualidade da água, como temperatura, salinidade, $\mathrm{pH}$ e oxigênio dissolvido não apresentaram diferenças significativas entre os tratamentos (Tabelas 1 e 2). O sistema de microcosmo manteve as variáveis físico-químicas basicamente idênticas para todos os tratamentos e dentro da faixa recomendada para o cultivo da espécie (Van Wyk \& Scarpa, 1999; Lin \& Chen, 2001, 2003; Decamp et al., 2007; Avnimelech, 2009; Furtado et al., 2011; Gaona et al., 2011; Maicá et al., 2012). Portanto o sistema de recirculação, baseado no esquema proposto por Moss $\&$ Moss (2004), mostrou-se eficiente para separar os efeitos do estresse populacional e da degradação da qualidade da água, sobre o desempenho dos camarões, em razão do aumento populacional.

Animais cultivados em sistemas de cultivo em biofloco frequentemente apresentam elevados índices de sobrevivência (Arnold et al., 2009). Porém, nos dois experimentos, as taxas de sobrevivência foram afetadas pelo aumento da densidade, tendo-se observado relação inversa entre o aumento da densidade e a redução da sobrevivência (Tabela 3 e 4).

No primeiro experimento, a densidade de 1.250 camarões por $\mathrm{m}^{3}$ obteve a maior sobrevivência, com média de $91,1 \pm 14,73 \%$, e não houve diferença significativa entre as densidades 833 e 1.667 camarões por $\mathrm{m}^{3}$. As menores sobrevivências ocorreram nos tratamentos com as densidades 2.083 e 2.500 camarões por $\mathrm{m}^{3}$, as quais não diferiram entre si. No segundo experimento, as menores sobrevivências - 63,1 e $54,1 \%$ - foram respectivamente as dos tratamentos com 1.388 e 1.667 camarões por $\mathrm{m}^{3}$. Os demais tratamentos apresentaram taxas de sobrevivência acima de 90,6\%. Os resultados corroboram os de diversos estudos de densidade de estocagem sobre diferentes espécies de peneídeos (Wasielesky Junior et al., 2001; Coman et al., 2004; Moss \& Moss, 2004; Arnold et al., 2009; Krummenauer et al., 2011).

Arnold et al. (2005) relataram que a falta de espaço viável gera estresse aos camarões e os leva ao canibalismo, fato observado no presente trabalho, tendo-se em vista que a qualidade de água não foi responsável pela mortalidade dos tratamentos com densidade mais elevada. Esta falta de espaço parece estar relacionada à capacidade de biomassa por metro cúbico que o camarão-branco suporta, a qual pode variar em cada sistema de cultivo. Nos dois experimentos, as maiores biomassas finais foram próximas $\left(9,92\right.$ e $10,77 \mathrm{~kg} \mathrm{~m}^{-3}$, respectivamente) e não comprometeram a sobrevivência, independentemente da densidade. Esta hipótese corrobora os resultados obtidos por Fóes et al. (2011)

Tabela 1. Média \pm desvio-padrão das variáveis físico-químicas de qualidade da água, registrados nos experimentos de cultivo de camarão-branco (Litopenaeus vannamei) cultivado a diferentes densidades em sistema de biofloco.

\begin{tabular}{|c|c|c|c|c|c|c|c|c|c|c|}
\hline \multirow[t]{2}{*}{ Parâmetro } & \multicolumn{5}{|c|}{ Experimento I / densidade (camarões por $\mathrm{m}^{3}$ ) } & \multicolumn{5}{|c|}{ Experimento II / densidade (camarões por $\mathrm{m}^{3}$ ) } \\
\hline & 833 & 1.250 & 1.667 & 2.083 & 2.500 & 556 & 833 & 1.111 & 1.388 & 1.667 \\
\hline Temperatura de manhã $\left({ }^{\circ} \mathrm{C}\right)$ & $27,50 \pm 0,782$ & $27,10 \pm 1,20$ & $28,10 \pm 0,91$ & $27,60 \pm 1,03$ & $26,70 \pm 2,01$ & $26,40 \pm 1,59$ & $25,70 \pm 2,03$ & $25,90 \pm 1,90$ & $26,10 \pm 1,61$ & $25,80 \pm 1,78$ \\
\hline Temperatura de tarde $\left({ }^{\circ} \mathrm{C}\right)$ & $29,20 \pm 1,302$ & $29,70 \pm 1,02$ & $28,30 \pm 1,27$ & $29,50 \pm 1,30$ & $29,10 \pm 1,87$ & $28,70 \pm 1,90$ & $27,90 \pm 1,34$ & $28,20 \pm 1,66$ & $28,10 \pm 1,70$ & $28,50 \pm 1,51$ \\
\hline $\mathrm{pH}$ & $7,32 \pm 1,22$ & $7,02 \pm 0,82$ & $7,16 \pm 0,71$ & $7,11 \pm 0,90$ & $7,27 \pm 1,03$ & $7,03 \pm 1,40$ & $7,21 \pm 0,80$ & $7,34 \pm 1,20$ & $7,56 \pm 0,70$ & $7,33 \pm 1,20$ \\
\hline Oxigênio dissolvido $\left(\mathrm{mg} \mathrm{L}^{-1}\right)$ & $4,70 \pm 1,51$ & $4,80 \pm 1,79$ & $4,51 \pm 2,01$ & $4,32 \pm 1,41$ & $4,30 \pm 1,30$ & $5,10 \pm 1,70$ & $5,80 \pm 1,92$ & $5,45 \pm 1,81$ & $5,07 \pm 1,45$ & $5,40 \pm 1,70$ \\
\hline
\end{tabular}


que, ao estudar o efeito de diferentes densidades $\left(1.388,2.778,4.166\right.$ e 5.556 camarões por $\left.\mathrm{m}^{3}\right)$ em pós-larvas de Farfantepenaeus paulensis, cultivadas no sistema bioflocos, não observaram diferenças significativas entre as médias de sobrevivência, que foram acima de $85,9 \%$, no entanto, o crescimento à maior densidade foi limitado.

No experimento I, ao final dos 40 dias de experimento, observou-se correlação negativa $(\mathrm{p}<0,05)$ da densidade de estocagem nos valores de peso final médio $\left(\hat{\mathrm{y}}=-2,6351 \mathrm{x}+7,88, \mathrm{R}^{2}\right.$ $=0,959)$, e os menores valores foram observados às densidades mais elevadas. Os pesos médios finais dos camarões cultivados às densidades $833,1.250$ e 1.667 camarões por $\mathrm{m}^{3}$ foram estatisticamente iguais, porém, houve deficit de crescimento às densidades de 2.083 e 2.500 camarões por $\mathrm{m}^{3}$. Isto já foi observado em diversas espécies de peneídeos,

Tabela 2. Média \pm desvio-padrão, valores máximos e mínimos dos parâmetros de qualidade da água, registrados no tanque-matriz dos experimentos de cultivo de camarão-branco (Litopenaeus vannamei) a diferentes densidades no sistema de biofloco.

\begin{tabular}{|c|c|c|c|c|c|c|}
\hline \multirow{2}{*}{ Parâmetro } & \multicolumn{3}{|c|}{ Experimento I } & \multicolumn{3}{|c|}{ Experimento II } \\
\hline & Média \pm desvio-padrão & Mínimo & Máximo & Média \pm desvio-padrão & Mínimo & Máximo \\
\hline Salinidade & $33,50 \pm 0,43$ & 32 & 36 & $34,00 \pm 0,70$ & 31 & 35 \\
\hline Secchi $(\mathrm{cm})$ & $17,80 \pm 3,20$ & 8,9 & 21,4 & $12,20 \pm 4,30$ & 7,1 & 18,7 \\
\hline Sólidos suspensos totais $\left(\mathrm{mg} \mathrm{L}^{-1}\right)$ & $223,00 \pm 94,81$ & 133 & 571 & $391,00 \pm 67,54$ & 147 & 501 \\
\hline Volume do floco $\left(\mathrm{ml} \mathrm{L}^{-1}\right)$ & $18,80 \pm 9,70$ & 16,2 & 41,4 & $27,10 \pm 10,30$ & 18,5 & 33,6 \\
\hline Amônia (N-AT mg L'-1) & $0,21 \pm 0,06$ & 0,09 & 0,71 & $0,32 \pm 0,12$ & 0,1 & 0,66 \\
\hline Nitrito $\left(\mathrm{N}-\mathrm{NO}_{2} \mathrm{mg} \mathrm{L}^{-1}\right)$ & $3,51 \pm 0,80$ & 1,39 & 8,91 & $4,22 \pm 1,30$ & 2,22 & 9,72 \\
\hline Nitrato $\left(\mathrm{N}-\mathrm{NO}_{3} \mathrm{mg} \mathrm{L}^{-1}\right)$ & $9,71 \pm 3,47$ & 4,71 & 15,22 & $11,31 \pm 4,31$ & 5,71 & 17,19 \\
\hline Fosfato $\left(\mathrm{P}-\mathrm{PO}_{4} \mathrm{mg} \mathrm{L}^{-1}\right)$ & $0,21 \pm 0,07$ & 0,1 & 0,98 & $0,32 \pm 0,09$ & 0,1 & 0,65 \\
\hline
\end{tabular}

Tabela 3. Média \pm desvio-padrão de peso final, sobrevivência, taxa de conversão alimentar, taxa de crescimento específico diário, biomassa final e densidade final de camarão-branco (Litopenaeus vannamei) cultivado às densidades de 833, 1.250, $1.667,2.083$ e 2.500 camarões por $\mathrm{m}^{3}$ em sistema de biofloco (experimento I).

\begin{tabular}{|c|c|c|c|c|c|}
\hline \multirow[t]{2}{*}{ Parâmetro } & \multicolumn{5}{|c|}{ Densidade (camarões por $\mathrm{m}^{3}$ ) } \\
\hline & 833 & 1.250 & 1.667 & 2.083 & 2.500 \\
\hline Peso médio final (g) & $7,53 \pm 0,73 a$ & $7,23 \pm 0,81 \mathrm{a}$ & $6,74 \pm 0,87 a$ & $6,12 \pm 1,05 b$ & $6,06 \pm 1,12 b$ \\
\hline Sobrevivência (\%) & $84,33 \pm 5,71 \mathrm{a}$ & $91,11 \pm 14,73 \mathrm{a}$ & $89,89 \pm 13,81 \mathrm{a}$ & $68,60 \pm 10,58 b$ & $62,96 \pm 11,10 b$ \\
\hline Taxa de conversão alimentar & $1,34 \pm 0,09 \mathrm{a}$ & $1,29 \pm 0,10 \mathrm{a}$ & $1,36 \pm 0,10 \mathrm{a}$ & $1,84 \pm 0,36 \mathrm{a}$ & $2,49 \pm 0,58 b$ \\
\hline Taxa de crescimento específico diário (\%) & $15,75 \pm 1,40$ & $15,00 \pm 1,64$ & $13,77 \pm 1,75$ & $12,22 \pm 2,10$ & $11,97 \pm 1,30$ \\
\hline Biomassa final $\left(\mathrm{kg} \mathrm{m}^{-3}\right)$ & $5,27 \pm 1,09 \mathrm{a}$ & $8,05 \pm 1,33 \mathrm{~b}$ & $9,92 \pm 1,97 b$ & $8,73 \pm 2,10 \mathrm{~b}$ & $9,52 \pm 2,83 \mathrm{~b}$ \\
\hline Densidade final (camarões por $\mathrm{m}^{3}$ ) & $700,00 \pm 36,10 \mathrm{a}$ & $1.113,50 \pm 113,6 b$ & $1.497,00 \pm 116,10 \mathrm{c}$ & $1.427,70 \pm 99,10 \mathrm{c}$ & $1.572,00 \pm 141,60 \mathrm{c}$ \\
\hline
\end{tabular}

${ }^{(1)}$ Médias seguidas de letras iguais, nas colunas, não diferem entre si, pelo teste de Tukey, a 5\% de probabilidade.

Tabela 4. Média \pm desvio-padrão de peso final, sobrevivência, taxa de conversão alimentar, taxa de crescimento específico diário, biomassa final e densidade final de camarão-branco (Litopenaeus vannamei) cultivado às densidades de 556, 833, $1.111,1.388$ e 1.667 camarões por $\mathrm{m}^{3}$ em sistema de biofloco (experimento II).

\begin{tabular}{|c|c|c|c|c|c|}
\hline \multirow[t]{2}{*}{ Parâmetro } & \multicolumn{5}{|c|}{ Densidade (camarões por $\mathrm{m}^{3}$ ) } \\
\hline & 566 & 833 & 1.111 & 1.388 & 1.667 \\
\hline Peso médio final (g) & $11,42 \pm 0,98 \mathrm{a}$ & $10,52 \pm 0,87 b$ & $10,64 \pm 1,07 b$ & $10,90 \pm 1,23 b$ & $10,01 \pm 1,67 b$ \\
\hline Sobrevivência (\%) & $90,63 \pm 3,23 a$ & $95,11 \pm 4,22 \mathrm{a}$ & $91,33 \pm 3,78 \mathrm{a}$ & $63,06 \pm 5,43 b$ & $54,06 \pm 3,42 \mathrm{c}$ \\
\hline Taxa de conversão alimentar & $1,23 \pm 0,1 \mathrm{a}$ & $1,17 \pm 0,1 \mathrm{a}$ & $1,34 \pm 0,28 \mathrm{a}$ & $1,71 \pm 0,23 b$ & $1,88 \pm 0,43 b$ \\
\hline Taxa de crescimento específico diário (\%) & $12,75 \pm 1,33$ & $10,5 \pm 1,03$ & $10,8 \pm 1,2$ & $11,45 \pm 1,38$ & $9,22 \pm 0,98$ \\
\hline Biomassa final $\left(\mathrm{kg} \mathrm{m}^{-3}\right)$ & $5,73 \pm 0,2 \mathrm{a}$ & $8,29 \pm 0,3 b$ & $10,77 \pm 0,4 \mathrm{c}$ & $9,53 \pm 0,30 \mathrm{c}$ & $9,01 \pm 0,40 \mathrm{bc}$ \\
\hline Densidade final (camarões por $\mathrm{m}^{3}$ ) & $505,5 \pm 25,01 \mathrm{a}$ & $788,5 \pm 41,57 b$ & $1.013,8 \pm 38,25 \mathrm{c}$ & $875,00 \pm 47,80 \mathrm{bc}$ & $900,00 \pm 33,88 \mathrm{bc}$ \\
\hline
\end{tabular}

${ }^{(1)}$ Médias seguidas de letras iguais, nas colunas, não diferem entre si, pelo teste de Tukey, a 5\% de probabilidade. 
como Litopenaeus stylirostris (Martin et al., 1998), Litopenaues setiferus (Williams et al., 1996), Fenneropenaeus indicus (Emmerson \& Andrews, 1981), Penaeus japonicus (Coman et al., 2004) e Penaeus monodon (Ray \& Chien, 1992).

Houve relação negativa entre aumento da densidade e o crescimento dos camarões, o que corrobora os resultados de diversos autores (Moss \& Moss, 2004; Arnold et al., 2009). A redução do crescimento e a sobrevivência de juvenis de peneídeos cultivados a altas densidades está relacionada a uma combinação de fatores, como a diminuição da viabilidade de espaço e a produtividade natural (Peterson \& Griffith, 1999), bem como a degradação da qualidade da água e o acúmulo de sedimento (Arnold et al., 2005). No presente estudo, a qualidade da água e a disponibilidade de alimento natural foram mantidas iguais para todos os tratamentos. Assim, as menores taxas de crescimento, provavelmente, foram consequência da limitação de espaço gerada pelas elevadas densidades utilizadas.

$\mathrm{O}$ tratamento à densidade de 833 camarões por $\mathrm{m}^{3}$ teve a menor biomassa $\left(5,27 \pm 1,09 \mathrm{~kg} \mathrm{~m}^{-3}\right)$, tendose diferenciado dos demais tratamentos, que não apresentaram diferença entre si. Além disso, os valores da taxa de conversão alimentar (TCA) de 1,29 a 2,49 podem resultar em custos financeiros significativos, tendo-se em vista que em muitos países a alimentação é o maior custo na produção do L. vannamei (Wasielesky Junior et al., 2006). Assim, a densidade de 1.667 camarões por $\mathrm{m}^{3}$ pode ser considerada a ideal para o cultivo de camarão-branco, com peso de 1 a 7 g, nas condições de cultivo descritas no presente trabalho.

No experimento II, os parâmetros de crescimento não apresentaram correlação com as diferentes densidades de estocagem. Todos os tratamentos apresentaram taxas de crescimento apropriadas (Tabela 4), e o maior peso médio final foi de 11,42 g à densidade 556 camarões por $\mathrm{m}^{3}$, com TCA semelhante à de diversos trabalhos de engorda do camarão-branco em sistema de biofloco (Wasielesky Junior et al., 2006; Krummenauer et al., 2011)).

A maior biomassa $\left(10,77 \pm 0,4 \mathrm{~kg} \mathrm{~m}^{-3}\right)$ foi detectada no tratamento à densidade 1.111 camarões por $\mathrm{m}^{3}$, e foi semelhante nos tratamentos às densidades de estocagem de 1.388 e 1.667 camarões por $\mathrm{m}^{3}$, em contraste com o tratamento a 566 camarões por $\mathrm{m}^{3}$, que apresentou a menor biomassa $\left(5,73 \pm 0,2 \mathrm{~kg} \mathrm{~m}^{-3}\right)$.
A relação inversa entre a densidade e o crescimento não foi tão acentuada como no primeiro experimento. Isto pode ter ocorrido pela alta mortalidade dos tratamentos 1.388 e 1.667 camarões por $\mathrm{m}^{3}$, que resultaram em densidades finais de 1.497 e 1.013 camarões por $\mathrm{m}^{3}$, respectivamente. Possivelmente, os camarões destes tratamentos apresentaram crescimento compensatório, como foi descrito por muitos autores (Ali et al., 2003; Oh et al., 2007) que relatam que, após um deficit de crescimento, os organismos podem apresentar "crescimento compensatório", quando cultivados em condições adequadas. Este aumento da taxa de crescimento é resultado de estratégias adotadas pelos organismos, tais como maior consumo de alimento no período pós-estresse (hiperfagia), aumento da eficiência alimentar, redução de gastos metabólicos e diminuição da locomoção (Ali et al., 2003).

Em geral, os resultados do presente trabalho podem auxiliar no dimensionamento e planejamento de unidades de cultivo, para potencializar a produtividade por meio do máximo aproveitamento do espaço disponível, com melhorias das técnicas de manejo.

\section{Conclusões}

1. O aumento da densidade de estocagem afeta o crescimento e a sobrevivência do camarão-branco cultivado em sistemas de biofloco.

2. A densidade de 1.667 camarões por $\mathrm{m}^{3}$ é mais eficiente para o cultivo de camarões com peso médio de 1 à $7 \mathrm{~g}$.

3. Para a fase de engorda, entre 6 e 12 gramas, a densidade ideal é de 1.111 camarões por $\mathrm{m}^{3}$.

\section{Agradecimentos}

Ao Conselho Nacional de Desenvolvimento Científico e Tecnológico (CNPq), ao Ministério da Pesca e Aquicultura (MPA) e à Coordenação de Aperfeiçoamento de Pessoal de Nível Superior (Capes), por bolsas e auxílio financeiro.

\section{Referências}

ALI, M.; NIECIEZA, A.; WOOTTON, R.J. Compensatory growth in fishes: a response to growth depression. Fish and Fisheries, v.4, p.147-190, 2003. DOI: 10.1046/j.1467-2979.2003.00120.x.

ARNOLD, S.J.; COMAN, F.E.; JACKSON, C.J.; GROVES, S.A. High-intensity, zero water-exchange production of juvenile tiger 
shrimp, Penaeus monodon: an evaluation of artificial substrates and stocking density. Aquaculture, v.293, p.42-48, 2009. DOI: 10.1016/j.aquaculture.2009.03.049.

ARNOLD, S.J.; SELLARS, M.J.; CROCOS, P.J.; COMAN, G.J. Response of juvenile brown tiger shrimp (Penaeus esculentus) to intensive culture conditions in a flow through tank system with three-dimensional artificial substrate. Aquaculture, v.246, p.231-238, 2005. DOI: 10.1016/j.aquaculture.2005.01.020.

AVNIMELECH, Y. Biofloc technology: a pratical guide book. Baton Rouge: The World Aquaculture Society, 2009. 182p.

BAUMGARTEN, M. da G.Z.; WALLNER-KERSANACH, M.; NIENCHESKI, L.F.H. Manual de análises em oceanografia química. 2.ed. Rio Grande: Universidade Federal de Rio Grande, 2010. 132p.

BOYD, C.E.; CLAY, J.W. Shrimp aquaculture and the environment. Scientific American, v.278, p.58-65, 1998. DOI: 10.1038/ scientificamerican0698-58.

BURFORD, M.A.; THOMPSON, P.J.; BAUMAN, R.H.; PEARSON, D.C. Nutrient and microbial dynamics in high-intensity, zero-exchange shrimp ponds in Belize. Aquaculture, v.219, p.393-411, 2003. DOI: 10.1016/S0044-8486(02)005-se-6.

COMAN G.J.; CROCOS, P.J.; PRESTON, N.P.; FIELDER, D. The effects of density on the growth and survival of different families of juvenile Penaeus japonicus Bate. Aquaculture, v.229, p.215-223, 2004. DOI: 10.1016/S0044-8486(03)00402-2.

DECAMP, O.; CONQUEST, L.; CODY, J.; FORSTER, I.; TACON, A.G.J. Effect of shrimp stocking density on size-fractionated phytoplankton and ecological groups of ciliated protozoa within zero-water exchange shrimp culture systems. Journal of the World Aquaculture Society, v.38, p.395-406, 2007. DOI: 10.1111/j.174 9-7345.2007.00111.x.

EMMERSON, W.D.; ANDREWS, B. The effect of stocking density on the growth, development and survival of Penaeus indicus-Milne Edwards larvae. Aquaculture, v.23, p.45-57, 1981. DOI: 10.1016/0044-8486(81)90006-5.

FÓES, G.K.; FRÓES, C.; KRUMMENAUER, D.; POERSCH, L.; WASIELESKY JUNIOR, W. Nursery of pink shrimp Farfantepenaeus paulensis in biofloc technology culture system: survival and growth at different stocking densities. Journal of Shellfish Research, v.30, p.367-373, 2011. DOI: 10.2983/035.030.0224.

FURTADO, P.S.; POERSH, L.H.; WASIELESKY JUNIOR, W. Effect of calcium hydroxide, carbonate and sodium bicarbonate on water quality and zootechnical performance of shrimp Litopenaeus vannamei reared in bio-flocs technology (BFT) systems. Aquaculture, v.321, p.130-135, 2011. DOI: 10.1016/j. aquaculture.2011.08.034.

GAONA, C.; POERSCH, L.H.; KRUMMENAUER, D.; FÓES, G.K.; WASIELESKY JUNIOR, W. The effect of solids removal on water quality, growth and survival of Litopenaeus vannamei in a biofloc technology culture system. International Journal of Recirculating Aquaculture, v.12, p.54-57, 2011.

KRUMMENAUER, D.; PEIXOTO, S.; CAVALLI, R.O.; POERSCH, L.; WASIELESKY JUNIOR, W. Superintensive culture of white shrimp, Litopenaeus vannamei, in a biofloc technology system in Southern Brazil at different stocking densities. Journal of the World Aquaculture Society, v.42, p.726-733, 2011. DOI: 10.1111/j.1749-7345.2011.00507.x.

KRUMMENAUER, D.; WASIELESKY JUNIOR, W.; CAVALLI, R.O.; PEIXOTO, S.; ZOGBI, P.B. Viabilidade do cultivo do camarão-rosa Farfantepenaeus paulensis (Crustácea: Decapoda) em gaiolas sob diferentes densidades durante o outono no Sul do Brasil. Ciência Rural, v.36, p.252-257, 2006. DOI: 10.1590/ S0103-84782006000100039.

LIN, Y.C.; CHEN, J.C. Acute toxicity of ammonia on Litopenaeus vannamei Boone juveniles at different salinity levels. Journal of Experimental Marine Biology and Ecology, v.259, p.109-119, 2001. DOI: 10.1016/S0022-0981(01)00227-1.

LIN, Y.C.; CHEN, J.C. Acute toxicity of nitrite on Litopenaeus vannamei (Boone) juveniles at different salinity levels. Aquaculture, v.224, p.193-201, 2003. DOI: 10.1016/ S0044-8486(03)00220-5.

MAICÁ, P.M.; BORBA, M.R. de; WASIELESKY JUNIOR, W. Effect of low salinity on microbial floc composition and performance of Litopenaeus vannamei (Boone) juveniles reared in a zero-water-exchange super-intensive system. Aquaculture Research, v.43, p.361-370, 2012. DOI: 10.1111/j.1365-2109.201 1.02838.x.

MARTIN, J.L.M.; VERAN, M.Y.; GUELORGET, O.; PHAM, D. Shrimp rearing: stocking density, growth, impact on sediment, waste output and their relationships studied through the nitrogen budget in rearing ponds. Aquaculture, v.164, p.135-149, 1998. DOI: 10.1016/S0044-8486(98)00182-3.

MOSS, K.R.K.; MOSS S.M. Effects of artificial substrate and stocking density on the nursery production of Pacific white shrimp Litopenaeus vannamei. Journal of World Aquaculture Society, v.35, p.536-542, 2004. DOI: 10.1111/j.1749-7345.2004.tb00121.x.

OH, S.-Y.; NOH, C.H.; CHO, S.H. Effect of restricted feeding regimes on compensatory growth and body composition of red sea bream, Pagrus major. Journal of World Aquaculture Society, v.38, p.443-449, 2007. DOI: 10.1111/j.1749-7345.2007.00116.x.

OTOSHI, C.A.; NAGUWA, S.S.; FALESCH, F.C.; MOSS, S.M. Shrimp behavior may affect culture performance at super-intensive stocking densities. Global Aquaculture Advocate, v.2, p.67-69, 2007.

PETERSON, J.J.; GRIFFITH, D.R. Intensive nursery systems. Global Aquaculture Advocate, v.2, p.60-61, 1999.

RAY, W.M.; CHIEN, Y.H. Effects of stocking density and aged sediment on tiger prawn, Penaeus monodon, nursery system. Aquaculture, v.104, p.231-248, 1992. DOI: 10.1016/0044-8486(92)90206-Z.

SOKAL, R.R.; ROHLF, F.J. Biometry: the principles and practices of statistics in biological research. San Francisco: W.H. Freeman, 1969. $776 \mathrm{p}$

VAN WYK, P.; SCARPA, J. Water quality and management. In: VAN WYK, P.; DAVIS-HODGKINS, M.; LARAMORE, R.; MAIN, K.L.; SCARPA, J. (Ed.). Farming marine shrimp in recirculating freshwater systems. Tallahassee: Department of Agriculture and Consumer Services, 1999. p.128-138.

Pesq. agropec. bras., Brasília, v.48, n.8, p.878-884, ago. 2013 DOI: $10.1590 / \mathrm{S} 0100-204 \mathrm{X} 2013000800010$ 
WASIELESKY JUNIOR, W.; ATWOOD, H.; STOKES, A.; BROWDY, C. Effect of natural production in a zero exchange suspended microbial floc based super-intensive culture system for white shrimp Litopenaeus vannamei. Aquaculture, v.258, p.396-403, 2006. DOI: 10.1016/j.aquaculture.2006.04.030.

WASIELESKY JUNIOR, W.; POERSCH, L.; JENSEN, L.; BIANCHINI, A. Effect of stocking density on growth of pen reared pink shrimp Farfantepenaeus paulensis (Pérez-Farfante, 1967) (Crustacea, Penaeidae). Nauplius, v.9, p.163-167, 2001.

WILlIANS, A.; DAVIS, D.; ARNOLD, C. Density-dependent growth and survival of Penaeus setiferus and Penaeus vannamei in a semi-closed recirculating system. Journal of the World Aquaculture Society, v.27, p.107-112, 1996. DOI: 10.1111/ j.1749-7345.1996.tb00600.x.

Recebido em 30 de dezembro de 2011 e aprovado em 31 de julho de 2013 